\title{
Elephant Endotheliotropic Herpesvirus Hemorrhagic Disease in Asian Elephant Calves in Logging Camps, Myanmar
}

Zaw Min Oo, Ye Htut Aung, Tin Tun Aung, Nyo San, Zaw Min Tun, Gary S. Hayward, Arun Zachariah

In recent years, an alarming number of cases of lethal acute hemorrhagic disease have occurred in Asian elephant calves raised in logging camps in Myanmar. To determine whether these deaths were associated with infection by elephant endotheliotropic herpesvirus (EEHV), we conducted diagnostic PCR subtype DNA sequencing analysis on necropsy tissue samples collected from 3 locations. We found that EEHV DNA from 7 PCR loci was present at high levels in all 3 calves and was the same EEHV1A virus type that has been described in North America, Europe, and other parts of Asia. However, when analyzed over 5,610 bp, the strains showed major differences from each other and from all previously characterized EEHV1A strains. We conclude that these 3 elephant calves in Myanmar died from the same herpesvirus disease that has afflicted young Asian elephants in other countries over the past 20 years.

Tn 1990, a brief description of a case of lethal acute hemorrhagic disease in a young Asian circus elephant in Switzerland that apparently involved a previously unknown herpesvirus was reported by Ossent et al. (1). However, it was not until the study of Richman et al. $(2,3)$, published in 1999, that this disease was shown to be associated with a novel herpesvirus designated elephant endotheliotropic herpesvirus (EEHV) 1 because of detection of nuclear inclusion bodies in damaged vascular endothelial cells in diseased heart and liver tissues. Those 2 studies provided evidence for the presence of high levels of small segments of the DNA genome of EEHV1 found by PCR sequencing

Author affiliations: University of Veterinary Sciences, Yezin,

Myanmar (Z.M. Oo, Y.H. Aung); Ministry of Environmental

Conservation and Forestry, Yangon, Myanmar (Z.M. Oo,

T.T. Aung, N. San, Z.M. Tun); Johns Hopkins School of Medicine, Baltimore, Maryland, USA (G.S. Hayward); Kerala Veterinary and Animal Sciences University, Wayanad, India (A. Zachariah)

DOI: https://doi.org/10.3201/eid2601.190159 techniques in blood samples and necropsy tissue samples from all major internal organs during active cases of hemorrhagic disease in 5 Asian elephant calves in zoos in North America and Europe, as well as in archival tissue block samples from 6 other cases in older elephants. Only 2 of the afflicted calves with less severe signs survived the acute disease after treatment with the human antiherpesvirus drug famciclovir; and viral DNA load in their blood was documented to decrease below levels of detection over a 4-6-week convalescence and recovery period. One young African elephant calf from a zoo that had a similar lethal hemorrhagic disease case had DNA from a second related virus species (EEHV2).

Since these original reports, numerous studies reviewed by Hayward (4) and Long et al. (5) have shown the presence of either of 2 chimeric subtypes of EEHV1A or EEHV1B in $\leq 50$ additional lethal cases and 10 drug-treated confirmed DNA-positive survivors with signs of disease in mostly young Asian elephants in Europe and North America (6-8). A small number of lethal and nonlethal cases of viral DNApositive hemorrhagic disease cases in young Asian elephants involved 2 additional related but considerably diverged species of Proboscivirus (EEHV4 and EEHV5) (9-16). EEHV4 is estimated to have last had common ancestors with EEHV1 35 million years ago and EEHV5 is estimated to have last had common ancestors with EEHV1 20 million years ago.

Furthermore, many examples of occasional shedding of these viruses, especially in trunk-wash and saliva samples have also been documented from adult Asian and African elephants with signs of disease (17-20; V.R. Pearson et al., Fox Chase Cancer Center, pers. comm., 2019 Jan 22). All currently available data $(5,13)$ support the concept that EEHV1A, EEHV1B, EEHV4, and EEHV5 are endemic in Asian elephants (Elephas maximus), whereas EEHV2, EEHV3, 

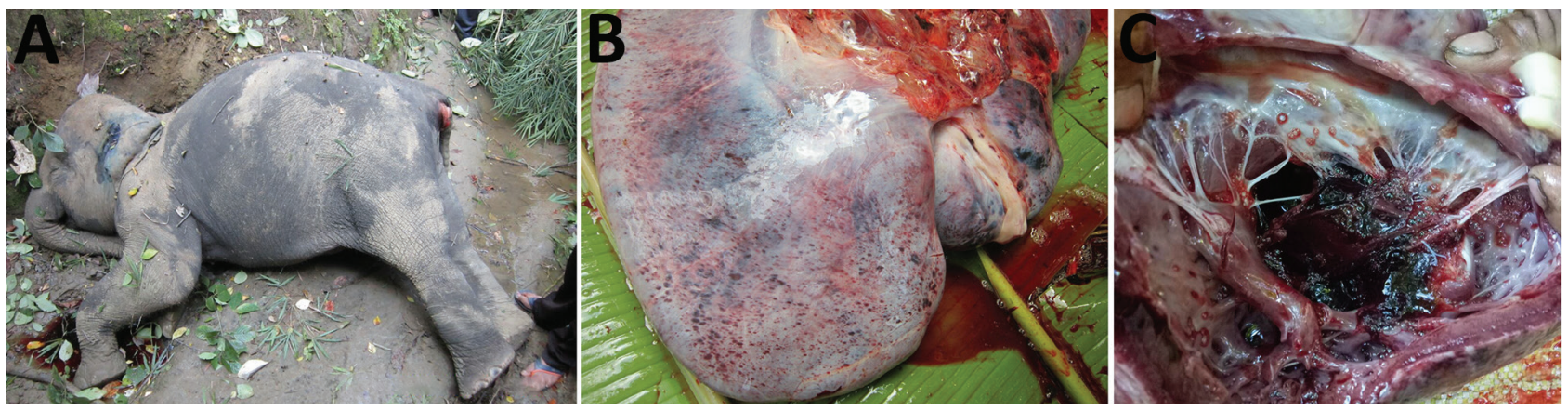

Figure 1. Gross morphologic characteristics of Asian elephant calf no. 3 (MP03) that had endotheliotropic herpesvirus hemorrhagic disease in logging camp, Myanmar. A) Before postmortem necropsy. B) Hemorrhagic lesions on intact surface of the liver.

C) Hemorrhagic lesions inside the atrium and valves of the dissected heart.

EEHV6, and EEHV7 are endemic to and largely ubiquitous in African elephants (Loxodonta africana). A subset of $\approx 20 \%$ of immunologically naive young Asian elephants 1-8 years of age are susceptible to the most severe forms of hemorrhagic disease that are caused predominantly by primary infections with the most pathogenic version of these viruses (EEHV1A).

A major aspect of the situation concerning possible long-term effects on breeding and survival of critically endangered Asian elephants worldwide is whether these viruses and the same hemorrhagic disease occur within countries in Asia and especially in wild populations. Reid et al. (21) reported an EEHV1 DNA-positive case from Cambodia. However, it was not until Zachariah et al. (22) published their results, after setting up a diagnostic PCR DNA laboratory in southern India to examine necropsy tissue collected from young orphan and wild elephants that suddenly died, that the range of the disease and virus became firmly established. These authors initially described 8 lethal cases of hemorrhagic disease associated with EEHV1A and 1 case associated with EEHV1B from India. Later in the same year, Sripiboon et al. (23) also reported 2 cases involving EEHV1A and EEHV4 from Thailand. More recent studies have also confirmed 2 lethal cases of EEHV1 hemorrhagic disease in Laos (24) and as many as 15 additional cases in Thailand $(25,26)$.

We have also expanded the studies in India to 22 lethal cases, including 12 in wild free-ranging calves (27). In this study, we report DNA sequenceconfirmed cases of lethal EEHV1A disease in 3 young logging camp elephants in Myanmar, including partial genetic analysis by PCR sequencing of the strains involved and comparison with all the cases from India and other representative cases worldwide.

\section{Materials and Methods}

Case 1 (M1) was in a 20-month-old, captive-born, female E. maximus calf that died suddenly on February 11, 2012, in Minbu District, Magway region, Myanmar. Case 2 (M2) was in a 22-month-old, captive-born, male E. maximus calf that died suddenly on September 14, 2013, in Nyaung Lay Pin District, Bago (East) region, Myanmar. Case 3 (M3) was in a 16-month-old, captiveborn, female E. maximus calf that died suddenly on January 8, 2014, in Nay Pyi Taw District, Myanmar.

We observed typical multiorgan hemorrhages, particularly in heart, liver, and peritoneum. We collected tissue samples and placed them in moleculargrade absolute ethanol during postmortem and stored them at $-20^{\circ} \mathrm{C}$. We used liver samples from each case for extraction of intracellular DNA by using the QIAamp Blood and Tissue Mini Kit (QIAGEN, https://www.qiagen.com). Conventional PCR amplifications were performed at the University of Veterinary Sciences (Yezin, Myanmar) during an EEHV workshop. These DNA samples were used as templates to amplify and sequence 7 standard preferred EEHV1 PCR DNA loci $(22,27)$ from all 3 cases, as well as an eighth locus (U51, vGPCR1) from 1 of these cases. We performed Sanger DNA sequencing and DNA sequence editing and constructed phylogenetic trees as described $(6,10,22)$. We used the updated sets of PCR primers reported by Zachariah et al. (27).

\begin{tabular}{|c|c|c|c|c|c|c|c|c|}
\hline \multirow[b]{2}{*}{ Case } & \multicolumn{8}{|c|}{ PCR gene locus } \\
\hline & E5 & vGPCR1 & U71-gM & POL-AB & TER & $\mathrm{gH}-\mathrm{TK}$ & HEL & vOX2 \\
\hline MP01, Myanmar1 & B1 & ND & A4 & A & $\mathrm{A} 1$ & D & A1 & 1 \\
\hline MP02, Myanmar2 & B1 & ND & A4 & A & $\mathrm{C}$ & D & $\mathrm{C} 2$ & 2 \\
\hline MP03, Myanmar3 & C1 & $\mathrm{E}$ & A5 & A & A3 & $\mathrm{D}$ & A2 & 3 \\
\hline
\end{tabular}

*EEHV, elephant endotheliotropic herpesvirus; ND, not determined; 1, identical to IP143 from India; 2, novel variant not seen previously; 3, identical to IP93 from India. 
Table 2. GenBank DNA file accession numbers for cases of hemorrhagic disease in 3 Asian elephant calves, Myanmar*

\begin{tabular}{lcccc}
\hline Case, virus code & Size, bp & MP01 & MP02 & MP03 \\
\hline E3(vGPCR5) & 962 & MF579041 & MF579042 & MF577043 \\
U38(POL) & 485 & MF579060 & MF579061 & MF579062 \\
U48(gH-TK) & 850 & MF464877 & MF464878 & MF464879 \\
U51(vGPCR1) & 677 & ND & MF & MF579097 \\
U60(TERex3) L & $741 / 724 / 726$ & MF579110 & MF579111 & MF579077 \\
U71-gM & 651 & MF579126 & MF579127 & MF579112 \\
U77(HEL) & $952 / 921 / 952$ & MF464889 & MF579128 \\
E54(vOX2-1) & 854 & MF464888 & & MF464890 \\
\hline${ }^{*}$ ND, not done. & & & &
\end{tabular}

We generated detailed results for genetic differences between EEHV DNA samples at each PCR locus as linear nucleotide-level polymorphisms by using Geneious software (https://www.geneious. com) from alignments made in Muscle software (https://www.drive5.com/muscle) or as Bayesian nearest neighbor-based phylogenetic trees at the DNA or protein level. For comparative purposes, we combined results for Myanmar (MP\#) with all data for samples from India (IP\#) and Sumatra (SP\#) evaluated
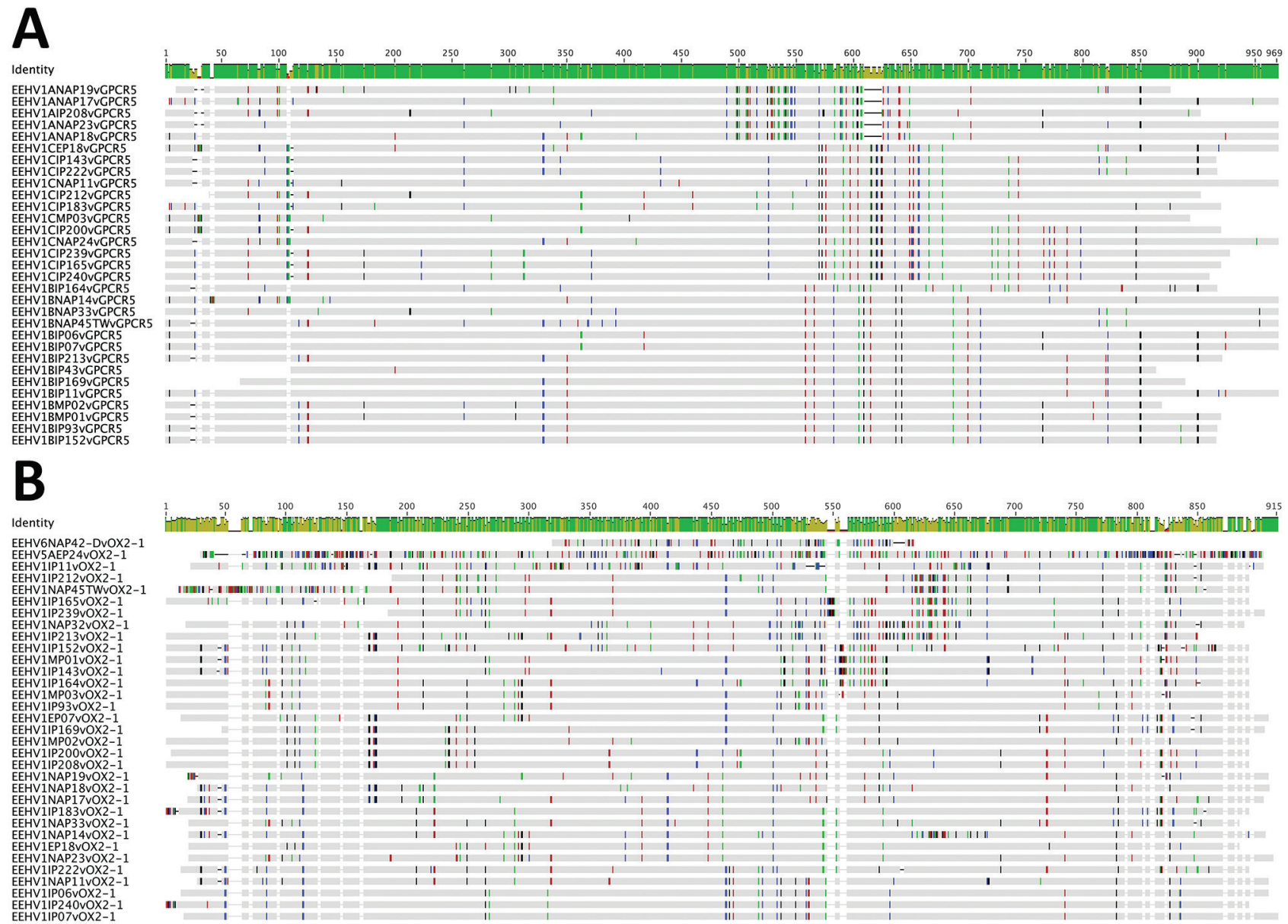

Figure 2. Nucleotide sequence polymorphism charts for Asian elephant calves that had endotheliotropic herpesvirus hemorrhagic disease in logging camp, Myanmar. Shown are comparisons for hemorrhagic disease cases MP01, MP02, and MP03 across 2 hypervariable EEHV1 PCR loci. A) E5(vGPCR5). B) E54(vOX2-1). Polymorphisms were generated by using Geneious (https://www. geneious.com) and MEGA5 (https://www.megasoftware.net) Bayesian phylogenetic trees comparing the Myanmar Proboscivirus case DNA sequence results with matching data available for all 22 cases from India (IP\#) and 2 cases from Sumatra (SP\#) plus several representative cases from North America (NAP\#) and Europe (EP\#) available in GenBank. Both prototype EEHV2(NAP12, Kijana) and EEHV5A(EP24, Vijay) genomes shown in the top lines were used as references in panel B, and EEHV1B(NAP19) was used as the reference for panel A. Assigned subtypes are included with designated code numbers listed on the left-hand side for each genome. Colored short vertical lines indicate single-nucleotide differences from the consensus sequences of all the genomes shown in each panel. Gaps or missing sequence segments appear as blank spaces. EEHV, elephant endotheliotropic herpesvirus. 
by our group. We also showed levels of divergence from prototype samples from North America (NAP\#) or Europe (EP\#) for each known subtype. We used other EEHV reference strains, such as EEHV1B, EEHV2, EEHV5, or EEHV6, as outgroups.

\section{Results}

We evaluated 3 cases of fatal hemorrhagic disease in captive-born E. maximus calves reared in camps in Myanmar. We provide gross morphology for 1 of the cases (Figure 1). The viral genomes in necropsy tissue DNA samples were designated MP01, MP02, and MP03 (for Myanmar Proboscivirus case numbers).
Overall results of gene subtyping PCR analyses (Table 1) and assigned GenBank accession numbers (Table 2) are provided.

At the time this study was conducted, we selected PCR primers provided to participants at a workshop in Myanmar after extensive previous analysis of 10 cases from Europe and 26 cases from North America as preferred standard sets that amplify 8 gene loci distributed across the 180-206-kb genomes of EEHV viruses. These geneloci included 4 well-conserved geneloci, U38(POL), U60(TERex3), U71/gM, and U77(HEL), which enabled identification of specific EEHV viruses present, as well as resolved chimeric EEHV1A subtype versus EEHV1B

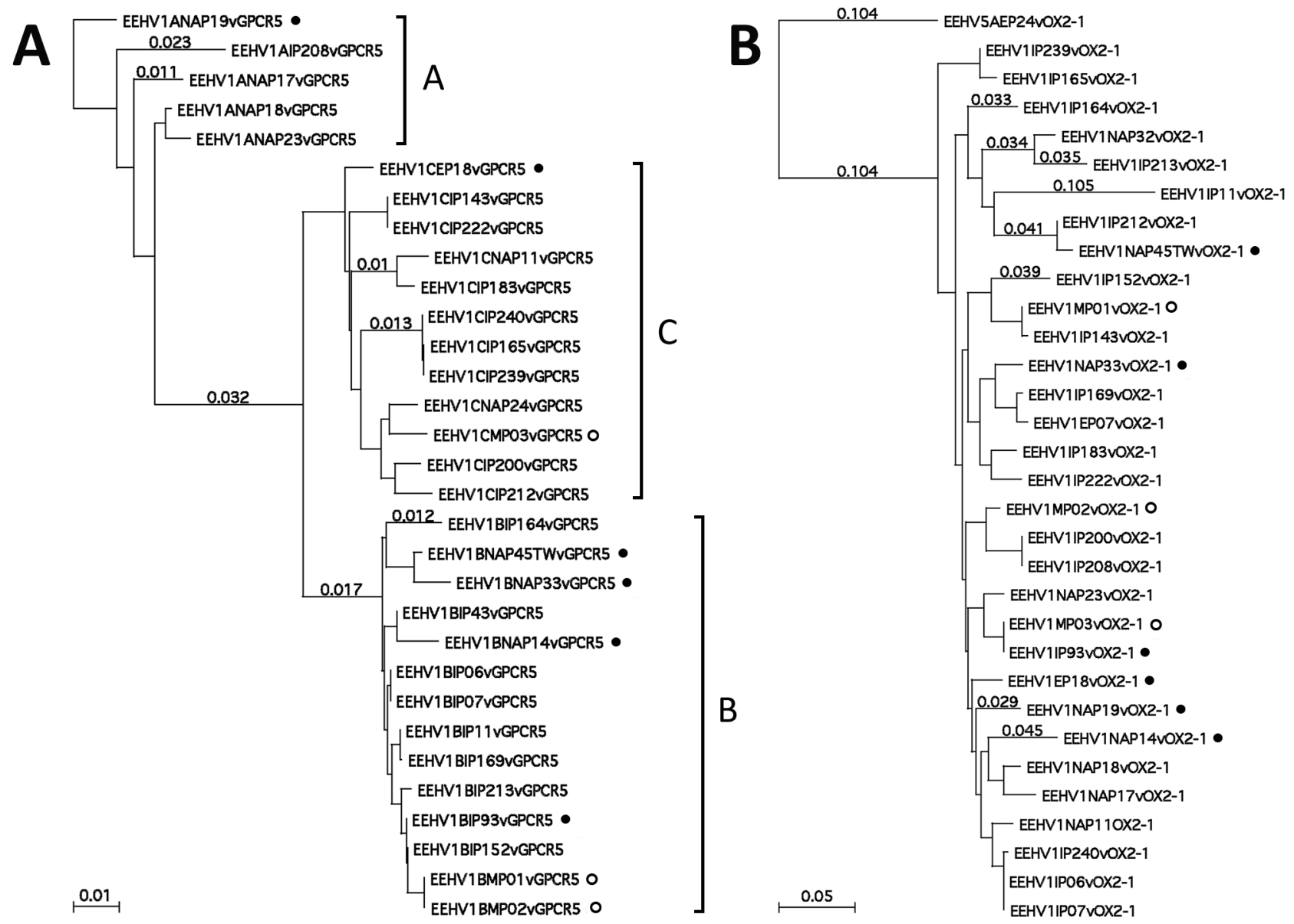

Figure 3. Protein level phylogenetic trees for Asian elephant calves that had endotheliotropic herpesvirus hemorrhagic disease in logging camp, Myanmar. Shown are comparison of examples of Asian EEHV1 at 2 representative hypervariable loci. A) EEHV1 E5(vGPCR5). B) E54(vOX2-1). Bayesian linear phylogenetic trees were generated from translated amino acid data in MEGA5.4.6 (https://www.megasoftware.net) by using similar aligned datasets as in Figure 2. Evolutionary history was inferred by using the maximum-likelihood method based on the Jones-Taylor-Thornton matrix-based model with the bootstrap consensus tree. Analysis in panel A involved $31 \mathrm{nt}$ sequences with 303 aa positions in the final dataset with EEHV1B(NAP19) as outgroup. Analysis in panel B involved 32 nt sequences with 244 aa positions in the final dataset and with EEHV5A(EP24) as outgroup. Numbers along branches are bootstrap values. Some representative branch length values are provided. The 3 major subtype clusters are indicated as A, B, or $C$ for E5(vGPCR5) along the right side of panel A, but no similar dramatic subtype clustering was discernable for E54(vOX2-1). All 6 examples in which the genomes have classic EEHV1B type core chimeric domain (CDI, CDII, and CDIII) features elsewhere are indicated by solid circles. Positions of the 3 cases from Myanmar are indicated by open circles. Scale bars indicate amino acid substitutions per site. EEHV, elephant endotheliotropic herpesvirus. 
Table 3. Nucleotide differences between EEHV strains from Asian elephant calves in Myanmar and EEHV1A and EEHV1B prototypes at the E5(vGPCR5) locus*

\begin{tabular}{|c|c|c|c|c|c|}
\hline vGPCR5 & MP01 & MP02 & MP03 & NAP23 & EP18 \\
\hline MP01 & - & 0 & 6.9 & 7.6 & 5.6 \\
\hline MP02 & 0 & - & 6.9 & 7.6 & 5.6 \\
\hline MP03 & 61 & 61 & - & 9.3 & 2.5 \\
\hline NAP23 & 67 & 66 & 83 & - & 7.9 \\
\hline EP18 & 50 & 50 & 22 & 71 & - \\
\hline
\end{tabular}

*Values to the right and above the dashes are percentages, and values to the left and below the dashes are number of nucleotides. EEHV, elephant endotheliotropic herpesvirus; EP18, prototype chimeric EEHV1B; NAP23, prototype EEHV1A.

subtype strains (6). In addition, we used 4 other PCR loci that encompass parts of well-characterized hypervariable genes. These loci were E5(vGPCR5), U48(gHTK), U51(vGPCR1), and E54(vOX2-1); we used them to address levels and patterns of individual variability for comparison with known, highly divergent, worldwide populations of EEHV1 strains.

We found that all 3 strains from Myanmar were EEHV1A variants that amplified strongly after the firstround conventional PCR. Weidentified nucleotide-level differences for 2 selected representative hypervariable PCR loci, namely E5(vGPCR5) and E54(vOX2-1) (Figure 2). Equivalent data for the U48(gH-TK) locus have already been reported for cases from India by Zachariah et al. (27). We provide 2 examples of the proteinlevel phylogenetic trees, again those for the E5(vGPCR5) and E54(vOX2-1) loci (Figure 3). The equivalent protein-level phylogenetic trees for U48(gH-TK) have been included in the report by Zachariah et al. (27). In addition, matching comparative DNA-level phylogenetic trees for all these samples at the 7 PCR loci other than U48(gH-TK) have been reported by Zachariah et al. (27). Finally, we report simple numerical difference comparisons (number and percentage) for the 3 strains from Myanmar with the prototype EEHV1A(NAP23, Kimba) and EEHV1B(EP18, Emelia) strains at the 3 most hypervariable loci, namely E5(vGPCR5), U48(gHTK), and E54(vOX2-1) (Tables 3-5).

Despite the consistent nucleotide divergence subtyping patterns, the 4 conserved loci evaluated rarely showed large numbers of amino acid polymorphisms. Even within the other 4 hypervariable loci, most nucleotide changes were also synonymous. In addition, unlike conserved loci, there are also numerous nonsynonymous amino acid changes, which provide additional robust supportive evidence for the same subtyping patterns being recognized in the trees at the DNA and protein levels. This finding is especially valid for the $\mathrm{U} 48(\mathrm{gH})$ protein, which is the only PCR segment evaluated here that is entirely encompassed within 1 of the EEHV1B chimeric domains (CD-II). The fact that the MP01 and MP02 versions of E5(vGPCR5) protein sequences are identical is a relatively rare exception, but otherwise the divergence of most EEHV1 strains from each other is evident in E5(vGPCR5) and E54(vOX2-1) protein phylogenetic trees (Figure 2), and similar but different gene-specific patterns were also found in U48(gH-TK) and U51(vGPCR1) protein trees.

The 3 cases from Myanmar were caused by distinct strains that showed major differences from each other, as well as from other cases in Asia, North America, and Europe that we evaluated. However, there were unusual similarities among these 3 cases. For example, MP01 and MP02 were in the same subtype groupings at 4 loci, including 2 conserved loci, namely U71-gM and U38(POL), as well as 2 hypervariable loci, namely E5(vGPCR5) and U48(gH)-TK, but showed different subtypes for the other 3 loci, namely U60(TERex3), U77(HEL), and E54(vOX2-1), whereas MP03 was distinguishable from MP01 and MP02 at all loci except U38(POL). Similar to many of the cases from India, MP03 was a subtype E in U51(vGPCR1) and had 28 differences (4.1\%) from the subtype A prototype EEHV1A(Kimba). MP01 and MP02 also had different subtypes (B1 for MP01 and C1 for MP02) from MP03 for E5(vGPCR5). Although all 3 cases from Myanmar had the same D subtype group within the U48(gH-TK) locus, MP03 still differed at this locus from the other 2 cases by $8 \mathrm{bp}$.

Although the captured E54(vOX2-1) gene is unusually well conserved when compared with the original host (African bush elephant [Loxodonta africana])

Table 4. Nucleotide differences between EEHV strains from Asian elephant calves in Myanmar and EEHV1A and EEHV1B prototypes at the U48(gH-TK) locus*

\begin{tabular}{lccccc}
\hline gH-TK & MP01 & MP02 & MP03 & NAP23 & EP18 \\
\hline MP01 & - & 0 & 1.0 & 8.7 & 28 \\
MP02 & 0 & - & 1.0 & 8.5 & 28 \\
MP03 & 8 & 8 & - & - & 28 \\
NAP23 & 73 & 73 & 71 & 233 & 28 \\
EP18† & 227 & 227 & 235 & - \\
\hline
\end{tabular}

*Values to the right and above the dashes are percentages, and values to the left and below the dashes are number of nucleotides. EEHV, elephant endotheliotropic herpesvirus; EP18, prototype chimeric EEHV1B; NAP23 prototype EEHV1A. 
Table 5. Nucleotide differences between EEHV strains from Asian elephant calves in Myanmar and EEHV1A and EEHV1B prototypes at the E54(vOX2-1) locus*

\begin{tabular}{lccccc}
\hline vOX-1 & MP01 & MP02 & MP03 & NAP23 & EP18 \\
\hline MP01 & - & 9.7 & 8.7 & 2.1 & 6.1 \\
MP02 & 82 & - & - & 3.7 & 5.7 \\
MP03 & 74 & 28 & 31 & - & 5.0 \\
NAP23 & 86 & 51 & 42 & 21 & 2.4 \\
EP18 & 87 & 48 & - & \\
\hline
\end{tabular}

*Values to the right and above the dashes are percentages, and values to the left and below the dashes are number of nucleotides.

EEHV, elephant endotheliotropic herpesvirus; EP18, prototype chimeric EEHV1B; NAP23, prototype EEHV1A.

version at the protein level, it shows the greatest DNAlevel divergence between all 3 strains from Myanmar and most other strains, in which MP01 differed from MP02 by 82 bp $(9.7 \%)$, MP02 from MP03 by 28 bp (3.3\%), and MP01 from MP03 by 74 bp (8.7\%). Nevertheless, MP01 and MP03 were identical at nt positions 280-480, and MP02 and MP03 differed by only $1 \mathrm{bp}$ over $>50 \%$ of the locus between nt positions 364-851.

We found that MP01 and MP03 were identical to another case from India (MP01 with IP143 and MP03 with IP93) at the otherwise hypervariable E54(vOX2-1) locus, whereas MP02 had unique features different from all other cases that we have evaluated worldwide. Overall, across all 4 core conserved loci, U38(POL), U71-gM, U60(TERex3), and U77(HEL), the differences from the prototype EEHV1A(Kimba) strain were no more than $15 \mathrm{nt}$ for MP01, $24 \mathrm{nt}$ for MP02, and $22 \mathrm{nt}$ for MP03. In contrast, the 3 most hypervariable loci showed a dramatically different pattern. In E5(vGPCR5), MP01 differed from Kimba by 67 (7.6\%), MP02 by 66 (7.7\%), and MP03by 83 (9.3\%) nt. In U48(gH-TK), MP01 differed from Kimba by 73 (8.7\%), MP02 by $73(8.7 \%)$, and MP03 by $71(8.5 \%)$ nt. Finally, in E54(vOX2-1), MP01 differed from Kimba by $86(10.1 \%)$, MP02 by 6651 $(6.1 \%)$, and MP03 by $31(3.7 \%)$ nt.

\section{Discussion}

The DNA sequence and subtyping results for the hemorrhagic disease cases in Myanmar showed that they involved different independent EEHV1A strains that were distinct from all other strains examined in countries in Asia or in zoos in Europe and North America. A similar pattern of variability was also obtained for 21 of the 22 cases in India, but this pattern was not always found. In contrast, in every situation observed in which 2 cases of hemorrhagic disease occurred at the same facility at nearly the same time (i.e., within days or weeks of each other), genomes were always identical at all loci tested. These results include paired cases at 2 facilities in Asia (India and Sumatra), 2 cases in Europe (United Kingdom and Germany), and $\geq 3$ cases in the United States (Texas, Florida, and Missouri). However, identical strains have not been reported for cases at different facilities (and different times), even in the same country. The fact that the 3 cases in Myanmar, although they showed some clear similarities across parts of the genomes and originated from within nearby geographic areas over a relatively short time frame, represent 3 distinct EEHV1A strains is not unexpected. However, more data from additional cases will be required to address whether overall populations of this virus in Myanmar have any common evolutionary features that differentiate them from the numerous and rather broadly diverged examples examined from India or Thailand.

Myanmar is now the sixth country in Asia (after Cambodia, Thailand, India, Laos, and Indonesia) in which apparent EEHV-associated hemorrhagic disease based on gross clinical or pathologic signs involving tissue hemorrhaging has been confirmed by PCR DNA subtype sequencing analysis. A brief preliminary speculation about these and multiple additional potential cases of similar hemorrhagic disease cases in Myanmar logging camps has been reported (28). Our findings will help with programs designed to address the increasing number of cases of lethal acute hemorrhagic disease in Asian elephants and possible longterm effects on breeding and survival of this critically endangered species.

\section{Acknowledgment}

We thank Sarah Y. Heaggans for providing valuable contributions for DNA sequence editing and generating phylogenetic trees.

This study was supported by a research grant to G.S.H. from the International Elephant Foundation.

\section{About the Author}

Dr. Oo is a veterinarian at the University of Veterinary Sciences, Yezin, Myanmar. His primary research interest is veterinary diseases, specifically diseases in elephants.

\section{References}

1. Ossent P, Guscetti F, Metzler AE, Lang EM, Rübel A, Hauser B. Acute and fatal herpesvirus infection in a young Asian elephant (Elephas maximus). Vet Pathol. 1990;27:131-3. https://doi.org/10.1177/030098589002700212

2. Richman LK, Montali RJ, Garber RL, Kennedy MA, Lehnhardt J, Hildebrandt T, et al. Novel endotheliotropic herpesviruses fatal for Asian and African elephants. Science. 1999;283: 1171-6. https://doi.org/10.1126/science.283.5405.1171 
3. Richman LK, Montali RJ, Cambre RC, Schmitt D, Hardy D, Hildbrandt $\mathrm{T}$, et al. Clinical and pathological findings of a newly recognized disease of elephants caused by endotheliotropic herpesviruses. J Wildl Dis. 2000;36:1-12. https://doi.org/10.7589/0090-3558-36.1.1

4. Hayward GS. Conservation: clarifying the risk from herpesvirus to captive Asian elephants. Vet Rec. 2012;170:202-3. https://doi.org/10.1136/vr.e1212

5. Long SY, Latimer EM, Hayward GS. Review of elephant endotheliotropic herpesviruses and acute hemorrhagic disease. ILAR J. 2016;56:283-96. https:/ / doi.org/10.1093/ilar/ilv041

6. Richman LK, Zong J-C, Latimer EM, Lock J, Fleischer RC, Heaggans SY, et al. Elephant endotheliotropic herpesviruses EEHV1A, EEHV1B, and EEHV2 from cases of hemorrhagic disease are highly diverged from other mammalian herpesviruses and may form a new subfamily. J Virol. 2014;88:13523-46. https://doi.org/10.1128/JVI.01673-14

7. Wilkie GS, Davison AJ, Watson M, Kerr K, Sanderson S, Bouts T, et al. Complete genome sequences of elephant endotheliotropic herpesviruses $1 \mathrm{~A}$ and $1 \mathrm{~B}$ determined directly from fatal cases. J Virol. 2013;87:6700-12. https:/ / doi.org/10.1128/JVI.00655-13

8. Ling PD, Reid JG, Qin X, Muzny DM, Gibbs R, Petrosino J, et al. Complete genome sequence of elephant endotheliotropic herpesvirus 1A. Genome Announc. 2013;1:e0010613. https://doi.org/10.1128/genomeA.00106-13

9. Garner MM, Helmick K, Ochsenreiter J, Richman LK, Latimer E, Wise AG, et al. Clinico-pathologic features of fatal disease attributed to new variants of endotheliotropic herpesviruses in two Asian elephants (Elephas maximus). Vet Pathol. 2009;46:97-104. https:/ / doi.org/10.1354/vp.46-1-97

10. Latimer E, Zong J-C, Heaggans SY, Richman LK, Hayward GS. Detection and evaluation of novel herpesviruses in routine and pathological samples from Asian and African elephants: identification of two new probosciviruses (EEHV5 and EEHV6) and two new gammaherpesviruses (EGHV3B and EGHV5). Vet Microbiol. 2011;147:28-41. https://doi.org/ 10.1016/j.vetmic.2010.05.042

11. Denk D, Stidworthy MF, Redrobe S, Latimer E, Hayward GS, Cracknell J, et al. Fatal elephant endotheliotropic herpesvirus type 5 infection in a captive Asian elephant. Vet Rec. 2012;171:380-1. https:// doi.org/10.1136/vr.e6833

12. Zong J-C, Latimer EM, Long SY, Richman LK, Heaggans SY, Hayward GS. Comparative genome analysis of four elephant endotheliotropic herpesviruses, EEHV3, EEHV4, EEHV5, and EEHV6, from cases of hemorrhagic disease or viremia. J Virol. 2014;88:13547-69. https:// doi.org/10.1128/JVI.01675-14

13. Zong J-C, Heaggans SY, Long SY, Latimer EM, Nofs SA, Bronson E, et al. Detection of quiescent infections with multiple elephant endotheliotropic herpesviruses (EEHVs), including EEHV2, EEHV3, EEHV6 and EEHV7, within lymphoid lung nodules or lung and spleen tissue samples from five asymptomatic adult african elephants. J Virol. 2015;90:3028-43. https:// doi.org/10.1128/JVI.02936-15

14. Wilkie GS, Davison AJ, Kerr K, Stidworthy MF, Redrobe S, Steinbach F, et al. First fatality associated with elephant endotheliotropic herpesvirus 5 in an Asian elephant: pathological findings and complete viral genome sequence. Sci Rep. 2014;4:6299. https:/ / doi.org/10.1038/srep06299

15. Ling PD, Long SY, Fuery A, Peng RS, Heaggans SY, Qin X, et al. A complete genome sequence of elephant endotheliotropic herpesvirus 4 (EEHV4): the first example of a GC-rich branch proboscivirus. MSphere. 2016;1:e00081-15. https:/ / doi.org/10.1128/mSphere.00081-15

16. Ling PD, Long SY, Zong JC, Heaggans SY, Qin X, Hayward GS. Comparison of the gene coding contents and other unusual features of the GC-rich and AT-rich branch probosciviruses. MSphere. 2016;1:e0091-16. https://doi.org/10.1128/ mSphere.00091-16

17. Stanton JJ, Zong J-C, Latimer E, Tan J, Herron A, Hayward GS, et al. Detection of pathogenic elephant endotheliotropic herpesvirus in routine trunk washes from healthy adult Asian elephants (Elephas maximus) by use of a real-time quantitative polymerase chain reaction assay. Am J Vet Res. 2010;71:925-33. https://doi.org/10.2460/ajvr.71.8.925

18. Stanton JJ, Zong J-C, Eng C, Howard L, Flanagan J, Stevens M, et al. Kinetics of viral loads and genotypic analysis of elephant endotheliotropic herpesvirus-1 infection in captive Asian elephants (Elephas maximus). J Zoo Wildl Med. 2013;44:42-54. https://doi.org/10.1638/1042-7260-44.1.42

19. Atkins L, Zong J-C, Tan J, Mejia A, Heaggans SY, Nofs SA, et al. EEHV-5, a newly recognized elephant herpesvirus associated with clinical and subclinical infections in captive asian elephants (Elephas maximus). J Zoo Wildl Med. 2013;44:136-43. https://doi.org/10.1638/1042-7260-44.1.136

20. Fuery A, Browning GR, Tan J, Long S, Hayward GS, Cox SK, et al. Clinical infection of captive Asian elephants (Elephas maximus) with elephant endotheliotropic herpesvirus 4. J Zoo Wildl Med. 2016;47:311-8. https:/ / doi.org/10.1638/2015-0072.1

21. Reid CE, Hildebrandt TB, Marx N, Hunt M, Thy N, Reynes JM, et al. Endotheliotropic elephant herpes virus (EEHV) infection. The first PCR-confirmed fatal case in Asia. Vet Q. 2006;28:61-4. https:/ / doi.org/10.1080/01652176.200 6.9695209

22. Zachariah A, Zong J-C, Long SY, Latimer EM, Heaggans SY, Richman LK, et al. Fatal herpesvirus hemorrhagic disease in wild and orphan Asian elephants in southern India. J Wildl Dis. 2013;49:381-93. https://doi.org/10.7589/2012-07-193

23. Sripiboon S, Tankaew P, Lungka G, Thitaram C. The occurrence of elephant endotheliotropic herpesvirus in captive Asian elephants (Elephas maximus): first case of EEHV4 in Asia. J Zoo Wildl Med. 2013;44:100-4. https://doi.org/10.1638/1042-7260-44.1.100

24. Bouchard B, Xaymountry B, Thongtip N, Lertwatcharasarakul P, Wajjwalku W. First reported case of elephant endotheliotropic herpes virus infection in Laos. J Zoo Wildl Med. 2014;45:704-7. https:/ / doi.org/ 10.1638/2013-0264R1.1

25. Lertwatcharasarakul $P$, Sanyathitiseree P, Thongtip N, Charoenphan P, Boonyasart B, Maneewan N, et al. Genetic variant of elephant endotheliotropic herpesvirus detected from captive Asian elephants (Elephas maximus) in Thailand from 2007 to 2013. Wetchasan Sattawaphaet. 2015;45:73.

26. Sripiboon S, Jackson B, Ditcham W, Holyoake C, Robertson I, Thitaram C, et al. Molecular characterisation and genetic variation of elephant endotheliotropic herpesvirus infection in captive young Asian elephants in Thailand. Infect Genet Evol. 2016;44:487-94. https:/ / doi.org/ 10.1016/j.meegid.2016.08.004

27. Zachariah A, Sajesh PK, Santhosh S, Bathrachalam C, Megha M, Pandiyan J, et al. Extended genotypic evaluation and comparison of twenty-two cases of lethal EEHV1 hemorrhagic disease in wild and captive Asian elephants in India. PLoS One. 2018;13:e0202438. https:/ / doi.org/10.1371/ journal.pone.0202438

28. Oo ZM. Health issues of captive Asian elephants in Myanmar. Gajah. 2012;36:21-2.

Address for correspondence: Gary S. Hayward, Viral Oncology

Program, Department of Oncology, Johns Hopkins School of Medicine, Baltimore, MD 21209, USA; email: ghayward@jhmi.edu 